\title{
Effets d'une intervention pédagogique visant l'apprentissage du contrôle inhibiteur sur le développement de prérequis liés à l'arithmétique chez les élèves du préscolaire âgés de 5 ans
}

\author{
Isabelle Deshaies $^{1^{\star}}$, Jean-Marie Miron $^{1}$ et Steve Masson ${ }^{2}$
}

\begin{abstract}
RÉSUMÉ
Les recherches en psychologie, en neurosciences cognitives et en didactique des mathématiques mènent à penser que trois prérequis sont importants dans l'apprentissage de l'arithmétique : le sens des nombres, la capacité d'établir des liens entre ce sens des nombres et les nombres symboliques, et la capacité de résister à l'utilisation de certaines stratégies inefficaces grâce au contrôle inhibiteur. Bien que des recherches aient déjà étudié les effets d'interventions pédagogiques visant le développement des deux premiers prérequis, aucune n'aurait évalué les effets d'une intervention visant les trois prérequis, ni les effets spécifiques du développement du contrôle inhibiteur. Pour cette raison, les effets d'une intervention ciblant les deux premiers prérequis et d'une autre ciblant les trois ont été évalués auprès de 126 élèves du préscolaire âgés de 5 ans. Les résultats montrent non seulement que les deux interventions ont des effets bénéfiques sur le développement de plusieurs habiletés numériques comparativement à un enseignement régulier, mais également qu'une intervention visant le contrôle inhibiteur, en plus des deux premiers prérequis, facilite davantage l'apprentissage du comptage et de la conservation du nombre. Cette étude met donc en évidence l'importance de développer le contrôle inhibiteur des élèves du préscolaire pour mieux les préparer à l'apprentissage de l'arithmétique.
\end{abstract}

\footnotetext{
${ }^{1}$ Département des sciences de l'éducation, Université du Québec à Trois-Rivières, Trois-Rivières, QC, Canada

${ }^{2}$ Département de didactique, Université du Québec à Montréal, Montréal, QC, Canada

* Correspondance avec l'auteure : isabelle.deshaies2@uqtr.ca
}

Pour citer cet article : Deshaies, I., Miron, J.-M. et Masson, S. (2020). Effets d'une intervention pédagogique visant l'apprentissage du contrôle inhibiteur sur le développement de prérequis liés à l'arithmétique chez les élèves du préscolaire âgés de 5 ans. Neuroéducation, 6(1), $49-64$.

DOI: https://doi.org/10.24046/neuroed.20200601.76

Reçu le 29 janvier 2019. Révision reçue le 18 septembre 2019.

Accepté le 9 novembre 2019. Publié en ligne le 6 juin 2020.

Neuroéducation, 6(1), 49-64

ISSN: 1929-1833

Tous droits réservés (C) 2020 - Association pour la recherche en neuroéducation 


\section{Introduction}

Les recherches montrent que 6 à 7 \% des élèves d'âge scolaire éprouvent de grandes difficultés en mathématiques (Charron, Duquesne, Marchand et Meljac, 2001; De Vriendt et Van Nieuwenhoven, 2010; Fuchs et Fuchs, 2005). Plusieurs de ces recherches indiquent que ce sont les élèves ayant des difficultés avec l'arithmétique élémentaire et les procédures de calcul chez qui les bases des mathématiques manquent le plus (Geary, Hoard et Bailey, 2012; Gersten, Jordan et Flojo, 2005). D'autres études montrent non seulement que les premiers apprentissages en mathématiques jouent un rôle important dans le fait d'éprouver éventuellement ou non des difficultés en mathématiques, mais également que les habiletés précoces en mathématiques constituent un important prérequis à la réussite éducative (Clark, Pritchard et Woodward, 2010; Duncan et al., 2007; Rourke et Conway, 1997). Ce caractère prédictif des habiletés précoces en mathématiques augmente la pertinence de mieux comprendre leurs déterminants, pour s'assurer d'intervenir adéquatement dès le préscolaire et ainsi favoriser à la fois le développement des compétences en mathématiques et la réussite éducative des élèves.

Plusieurs chercheurs associent les difficultés d'apprentissage des élèves en mathématiques à des notions issues du champ de l'arithmétique (Geary et al. 2012; Gersten et al., 2005). Ces élèves seraient également ceux qui sont plus lents dans les tâches élémentaires nécessitant des procédures mathématiques comme la lecture des nombres, la comparaison des nombres, la récitation d'une séquence de nombres et le dénombrement (Landerl, Bevan et Butterworth, 2004), de même que dans les tâches qui requièrent la manipulation de quantité de nombres (dénombrement et comparaison) (Rousselle et Noël, 2007) et la subitisation de petites quantités numériques (reconnaître globalement des quantités de un à quatre) (Koontz, 1996). Bien que ces recherches aient porté sur des élèves ayant des difficultés d'apprentissage en mathématiques au primaire, il n'en demeure pas moins que ces notions sont importantes pour tous les élèves et elles devraient conséquemment être vues et enseignées à tous dès le préscolaire.

Étant donné le rôle central de ces notions dans l'apprentissage des mathématiques, et plus spécifiquement de l'arithmétique, il convient de se questionner sur les pratiques d'enseignement les plus efficaces pour faciliter l'apprentissage de ces notions. À ce sujet, il est intéressant d'examiner les études en psychologie, en neurosciences cognitives et en didactique des mathématiques ayant ciblé cette question. Deshaies, Miron et Masson (2015) ont effectué une recension des écrits établissant des liens entre le fonctionnement cérébral et l'apprentissage de l'arithmétique afin de déterminer quels pourraient être les prérequis essentiels en mathématiques. D'après ces chercheurs, trois prérequis seraient essentiels à l'apprentissage des mathématiques, soit le développement du sens des nombres (reconnaître globalement, sans avoir recours au comptage, l'ensemble qui a le plus d'éléments lors d'une tâche de comparaison), l'établissement de liens entre ce sens des nombres et les nombres symboliques (associer la quantité à son nombre) et le développement du contrôle inhibiteur.

Comme le mentionne Dehaene (2011), le sens des nombres permet de faire la distinction entre deux quantités non symboliques et de déterminer laquelle est supérieure ou inférieure à l'autre; ce serait la base de la construction des compétences en mathématiques. De plus, les recherches de Mussolin, Mejias et Noël (2010) et de Piazza et al. (2010) ont permis de constater que l'acquisition des nombres symboliques peut améliorer le sens de l'approximation des nombres et ainsi permettre de comparer des quantités numériquement rapprochées sans avoir besoin de les compter. En ce sens, Dehaene (2011) soutient également que, pendant les années préscolaires, l'établissement d'un dialogue bidirectionnel entre le sens des nombres et le système des nombres symboliques conduit à un système où chaque symbole numérique est automatiquement attaché à un sens précis. L'établissement de liens entre le sens des nombres et les nombres symboliques serait donc une étape charnière dans l'apprentissage des mathématiques et un prérequis pour un passage réussi vers le $1^{\mathrm{er}}$ cycle du primaire (Ibid.). De plus, comme l'ont démontré les recherches de Houdé et al. (2000) et de Lubin, Lanoë, Pineau et Rossi (2012), apprendre des nouvelles notions n'implique pas seulement d'acquérir de nouvelles connaissances, mais également d'apprendre à bloquer certaines stratégies de raisonnement inefficaces grâce au contrôle inhibiteur. Une étude en neuroimagerie montre d'ailleurs que le contrôle inhibiteur serait central à certains apprentissages en mathématiques; par exemple, la tâche de conservation du nombre décrite dans l'article de Houdé et al. (2011).

Malgré l'importance présumée de ces prérequis dans l'apprentissage de l'arithmétique, il est étonnant de constater qu'aucune recherche n'ait tenté jusqu'à présent d'évaluer les effets d'une intervention pédagogique visant le développement de ces trois prérequis. En effet, une recension d'écrits de Deshaies, Miron, Picard et Masson (2020) a permis d'identifier que, sur un total de 22 programmes ou outils d'intervention visant le développement de l'un ou l'autre des prérequis, aucun ne vise explicitement le développement des trois prérequis. En effet, bien que plusieurs programmes d'intervention en mathématiques au préscolaire touchent le sens des nombres (Clements, DiBiase et Sarama, 2004; Griffin, 2004; Piazza et al., 2004; Wilson et al., 2006) ou le lien entre le sens des nombres et la représentation symbolique des nombres (Baroody, Eiland et Thompson, 2009; Baroody, Eiland, Purpura et Reid, 2012; Clements et Sarama, 2008; Clements, Sarama, Wolfe et Spitler, 2013; Griffin, 2004), aucun ne cible le développement explicite du contrôle inhibiteur, c'est-à-dire la capacité à contrôler ou bloquer certains automatismes de la pensée pouvant nuire au raisonnement numérique. Cependant, Houdé et al. (2011) et Lubin et al. (2012) ont développé et mis à l'épreuve un dispositif didactique (attrape-piège) pour aider 
les élèves à contrer leurs stratégies erronées (inhibition). Ces recherches ne ciblaient toutefois pas spécifiquement des élèves d'âge préscolaire ni le développement de compétences en mathématiques.

Ainsi, afin d'en savoir plus sur le rôle du développement du contrôle inhibiteur dans l'apprentissage d'habiletés numériques de base, deux interventions ont été créées. La première intervention, qui s'inspire des interventions existantes citées précédemment, cible les deux premiers prérequis (sens des nombres et le lien entre ce sens des nombres et le nombre symbolique) et la deuxième vise les trois prérequis (sens des nombres, le lien entre ce sens de nombres et le nombre symbolique et le contrôle inhibiteur). Cette étude vise, d'une part, à déterminer les effets de chacune des deux interventions (comparativement à un enseignement régulier en classe préscolaire 5 ans) sur des habiletés numériques de base (par exemple le traitement des quantités exprimées par les codes numériques analogique, arabe, et oral ainsi que de leurs liens avec les représentations numériques mentales). D'autre part, elle vise à évaluer les effets spécifiques de l'intervention visant le développement du contrôle cognitif en la comparant à l'intervention visant seulement les deux premiers prérequis.

En somme, les objectifs de cette recherche sont de :

1. comparer l'efficacité d'une intervention, au préscolaire, visant l'acquisition de deux prérequis mathématiques : le sens des nombres et le lien entre le sens des nombres et le nombre symbolique, à un enseignement régulier des mathématiques;

2. comparer l'efficacité d'une intervention, au préscolaire, visant l'acquisition de deux prérequis mathématiques : le sens des nombres, le lien entre le sens des nombres et le nombre symbolique, ainsi que le contrôle inhibiteur, à un enseignement régulier des mathématiques;

3. comparer l'efficacité d'une intervention, au préscolaire, visant l'acquisition de deux prérequis mathématiques : le sens des nombres et le lien entre le sens des nombres et le nombre symbolique, à une intervention visant plutôt l'acquisition de deux prérequis mathématiques: le sens des nombres, le lien entre le sens des nombres et le nombre symbolique, ainsi que le contrôle inhibiteur.

\section{Méthodologie}

\subsection{Participants}

Létude s'est déroulée dans la région du Centre-du-Québec, plus précisément dans la Commission scolaire de la Riveraine. Lors de celle-ci, nous avons ciblé des écoles provenant de milieux moyens sur le plan socioéconomique, cotés entre 5 et 8 selon lindice de défavorisation du MELS ${ }^{1}$, d'une même région.
Parmi ces écoles, les enseignants devaient être en poste à plus de 80 \% de leur tâche d'enseignement et non en remplacement. Ils ne devaient pas faire partie d'un projet particulier en mathématique pouvant venir influencer les facteurs à l'étude. Les classes ne respectant pas un ou l'autre des critères de sélection n'ont pu participer au projet de recherche.

De plus, afin d'obtenir une puissance statistique de 0,95 dans nos analyses projetées, nous avons procédé à une analyse G Power $^{2}$ afin de connaitre le nombre de participants nécessaire; le résultat de cette analyse indique 96 participants. La présente étude a recruté 126 participants, ce qui est au-delà du nombre requis pour l'analyse. La moyenne d'âge des élèves participants était de 5,2 ans. Les classes ont été distribuées au hasard à chacune des interventions.

\subsection{Interventions}

Les deux interventions, créées et destinées à une clientèle préscolaire, ont la même durée (quatre interventions de 15 minutes par semaine pendant cinq semaines), le même intervenant; soit l'enseignant en poste, la même structure (consignes de la tâche, exemples de la tâche, modélisation avec l'attrape-réponse ou l'attrape-piège et activités) et sont fondées sur les mêmes principes pédagogiques :

(1) les notions mathématiques sont présentées du simple au complexe;

(2) la planification inclut des rappels sur les connaissances antérieures (une fois l'activité enseignée, l'enseignant demande systématiquement aux élèves s'ils ont déjà fait une tâche semblable et l'expliquer);

(3) une rétroaction est systématiquement donnée aux participants (dès que la réponse est incorrecte, l'enseignant arrête l'activité, donne la bonne solution ainsi que la bonne stratégie et ensuite l'activité reprend), et

(4) les activités proposées sont espacées dans le temps (une fois une notion vue, elle est revue sous forme de rappel deux activités plus loin, puis cinq et dix). La première vise le développement des deux premiers prérequis (sens du nombre et liens entre le sens du nombre et les nombres symboliques) et la deuxième vise quant à elle les deux mêmes prérequis en plus du développement du contrôle inhibiteur.

Afin de travailler les deux premiers prérequis, les deux interventions s'appuient sur les recherches en didactique des mathématiques. Le sens des nombres est travaillé à partir de trois activités portant sur celui-ci (Ansari, 2008; Ansari, Dhital et Siong, 2006; Nosworthy et al., 2013) et deux en lien avec la subitisation perceptuelle et conceptuelle (Clements, 2007; Gallistel et Gelman, 1992; Kaufman, Lord, Reese et Volkmann, 1949). Le lien entre le sens des nombres et le nombre symbolique est travaillé à partir de 15 activités portant sur le dénombrement, la conservation du nombre, la comparaison des nombres, lacquisition du système arabe et la résolution de problèmes (Baruk, 2003; Bideaud et Lehalle, 2002; Gelman, 1978; Grégoire et Van Nieuwenhoven, 1995; Noël, 2005;

\footnotetext{
${ }^{1}$ www.mels.gouv.qc.ca/.../stat Indices defavorisation_2013 2014.pdf $\quad{ }^{2}$ http://www.gpower.hhu.de/
} 
Sophian, 1998). Pour plus de détails concernant les différentes activités, voir Deshaies (2017).

Le développement du contrôle inhibiteur est développé dans la deuxième intervention non pas à partir d'activités supplémentaires, mais à l'aide de dispositifs pédagogiques légèrement différents pour certaines activités et inspirées des travaux de Rossi, Lubin, Lanoë et Pineau (2012). Ces dispositifs didactiques sont utilisés seulement lorsque le jeu présenté aux élèves nécessite le contrôle inhibiteur. Par exemple, dans les activités liées à la conservation du nombre, alors que l'intervention 1 (sans contrôle inhibiteur) implique l'utilisation d'un « attrape-réponse » conçu pour amener les élèves à identifier les bonnes et les mauvaises réponses dans les énoncés présentés (voir figure 1), lintervention 2 (avec contrôle inhibiteur) implique plutôt l'utilisation d'un «attrape-piège » conçu pour amener les élèves à identifier non seulement les bonnes et les mauvaises réponses, mais aussi les réponses qui constituent des pièges, c'est-à-dire des réponses qui semblent intuitivement correctes, mais qui sont erronées (voir figure 1). Une alerte émotive (indiquant de faire attention, car il y a un piège dans l'activité) a été incluse dans l'intervention 2 (avec contrôle inhibiteur).

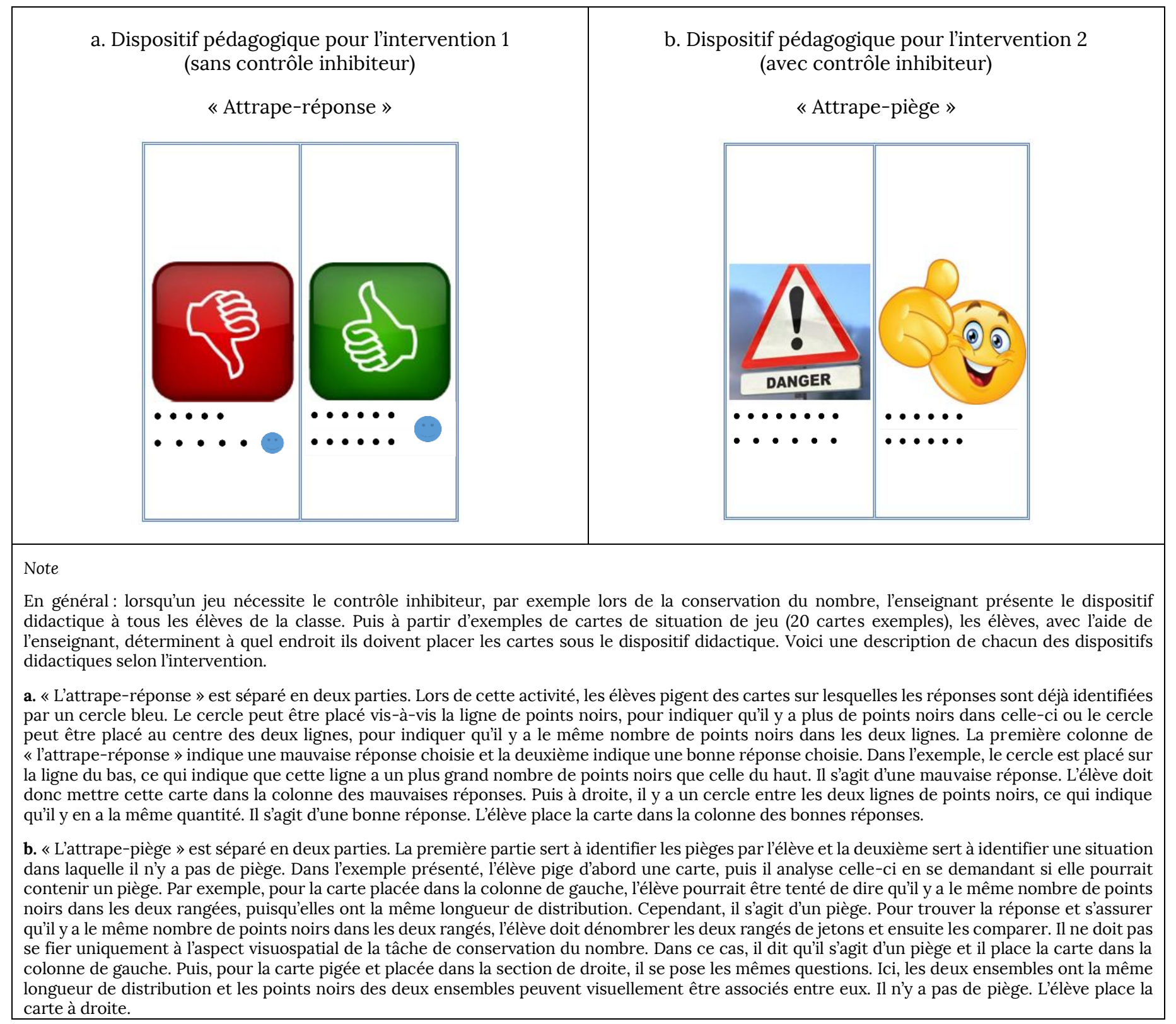

Figure 1. Dispositifs pédagogiques utilisés dans chacune des deux interventions 
Les deux interventions sont réalisées à partir de jeux mathématiques. Cette caractéristique est en partie due à la nécessité de respecter la philosophie du programme d'études des participants à cette recherche (Programme de formation de l'école québécoise, PFÉQ) (MELS, 2003) qui place le jeu au cœur de la pédagogie préscolaire. En ce sens, la recherche en neurosciences de Kohn (2004) mentionne que lorsque la tâche proposée permet aux élèves d'être engagés et motivés, ces derniers ressentent un stress minimal, ce qui leur permet d'atteindre des niveaux plus élevés de cognition. Ces élèves font davantage de connexions cérébrales ce qui leur permet de mieux maitriser la matière à l'étude. C'est ce qui est susceptible de se produire en contexte de jeu comme celui-ci.

En plus d'être comparées l'une par rapport à l'autre, les deux interventions sont comparées à un enseignement régulier tel que prescrit par le PFÉQ. Ce programme offre des orientations générales pour mettre en œuvre différentes activités en lien avec les nombres, sans toutefois préciser les apprentissages explicitement visés, laissant ainsi une grande marge de manœuvre aux enseignantes et enseignants. En ce sens, par des activités ludiques et quotidiennes, l'élève recevant un enseignement régulier réalise des activités visant à développer huit types de connaissances mathématiques, soit : les jeux de nombres, le dénombrement, l'association, la comparaison, le regroupement et la classification, la régularité, l'estimation et la mesure. Bien que mentionnées dans le PFÉQ, ces huit connaissances ne sont pas explicitement développées dans ce programme et laissent ainsi beaucoup d'ambiguité de la part des enseignants.

Les interventions se sont déroulées pendant cinq semaines durant les mois d'octobre et novembre. La passation des prétests a été réalisée par l'équipe de recherche la semaine précédant la mise en place de l'intervention et la passation des posttests a été réalisée par l'équipe de recherche la semaine suivant la fin de l'intervention. Afin de s'assurer de l'uniformité de la passation des tests ainsi que sa validité, l'équipe de recherche a reçu une formation de trois heures.

a. Consigne : Fais un trait sur l'ensemble ayant la plus grande valeur.

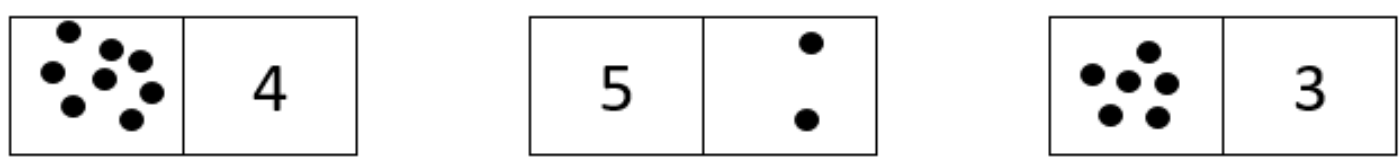

b. Consigne : Fais un trait sur l'ensemble ayant la plus grande valeur.
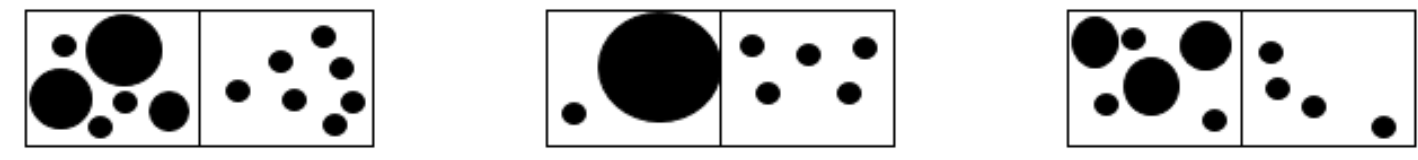

c. Consigne : Fais un trait sur l'ensemble ayant la plus grande valeur.
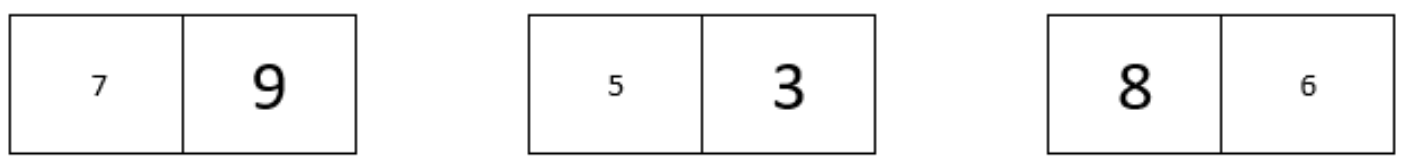

d. Consigne : Encercle l'ensemble s'il y a le même nombre de points dans les deux rangées. Si ce n'est pas égal, fais un trait sur la rangée de points où il $\mathrm{y}$ en a le plus grand nombre.
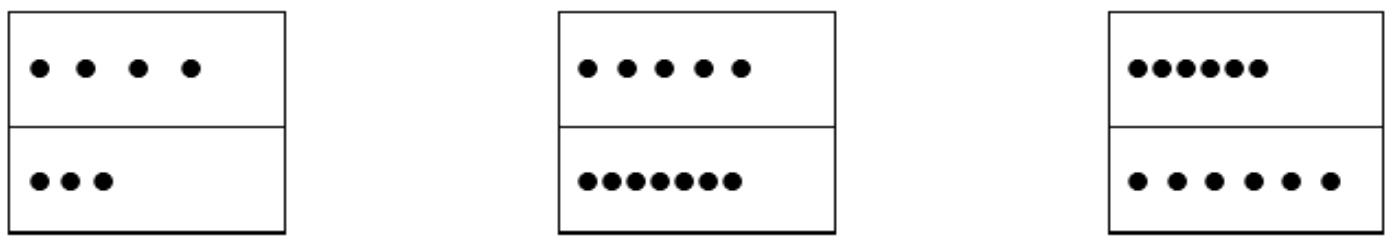

Note

L'exemple « a » permet de mesurer la capacité de comparer des nombres symboliques et non symboliques entre eux. L'exemple «b » permet de mesurer la capacité d'inhiber la taille des points pour les comparer entre eux. L'exemple «c » permet de mesurer la capacité d'inhiber la taille des chiffres pour pouvoir les comparer entre eux. L'exemple « d » permet de mesurer la capacité d'inhiber la longueur de la répartition des points pour pouvoir déterminer s'il y a égalité entre les deux ensembles.

Figure 2. Exemples de tests numériques nécessitant la capacité d'inhiber 


\subsection{Instruments}

Nous avons utilisé le Numeracy Screener non symbolique et symbolique (Nosworthy et al., 2013) pour mesurer l'habileté mathématique liée au sens des nombres et à la comparaison de nombres symboliques, le Tedi-Math (Van Nieuwenhoven, Grégoire et Noël, 2005) pour mesurer les compétences de base en mathématiques, ainsi que des tests développés par notre équipe de recherche. Les tests développés sont construits selon le modèle du Numeracy Screener, soit 56 items en tout, 14 items par page dont 7 de type intuitifs et 7 de type non-intuitifs nécessitant la capacité d'inhiber. Le score de ces tests est calculé en considérant le nombre d'items réussis sur le nombre d'items complétés. Le premier de ces tests permet de mesurer l'habileté à faire le lien entre le nombre symbolique et le nombre non symbolique en demandant à l'élève de faire un trait sur le plus grand des deux nombres, qu'il soit symbolique ou non-symbolique (voir figure 2a). Les trois autres tests ont été élaborés par notre équipe pour mesurer la capacité d'inhiber en contexte numérique des élèves du préscolaire. Ceux-ci demandent à l'élève d'inhiber la taille des points (voir figure $2 b$ ), la taille des chiffres (voir figure 2c) ou la longueur d'une rangée de points (voir figure 2d) pour comparer deux ensembles. Les tests conçus par l'équipe de recherche ont été mis à l'épreuve auprès d'enfant de 5 ans, mais n'ont toutefois pas encore été validés statistiquement.

\subsection{Design de recherche}

Le protocole de recherche utilisé est celui des quatre groupes de Solomon (Solomon, 1949). Ce design est particulièrement pertinent dans le cadre d'une recherche quasi expérimentale et permet d'examiner à la fois les principaux effets de l'intervention auprès des élèves et l'interaction de la passation du prétest sur le posttest auprès de ceux-ci. Par conséquent, la généralisation des résultats est étudiée de façon explicite dans ce protocole (Bégin et Ladouceur, 1980).

Les huit groupes classes ont été distribués aléatoirement selon le protocole de Solomon (voir tableau 1). Deux groupes ont expérimenté la première intervention visant le développement du sens du nombre et du lien entre ce sens des nombres et les nombres symboliques, deux groupes ont fait l'expérience de la deuxième intervention visant le sens des nombres, le lien entre ce sens des nombres et les nombres symboliques et le contrôle inhibiteur, et quatre groupes ont reçu un enseignement régulier selon le PFÉQ. En plus d'être distribués aléatoirement dans l'une ou l'autre des interventions, les groupes furent distribués aléatoirement pour la passation ou non des prétests. Ce design de recherche est représenté au tableau 1.

\subsection{Analyses}

Une fois les tests administrés aux élèves, nous avons procédé à l'analyse des résultats. Les analyses statistiques, effectuées à l'aide du logiciel SPSS (v.20), visent à déterminer (1) l'efficacité de l'intervention avec et sans contrôle inhibiteur comparativement à un enseignement régulier;
(2) l'efficacité d'une intervention avec contrôle inhibiteur versus sans contrôle inhibiteur; (3) l'effet de la passation du prétest chez les élèves du préscolaire. Pour ce faire, nous avons réalisé une analyse de variance (ANOVA) pour observer s'il y avait des différences statistiquement significatives auprès des groupes. Suite à celle-ci, nous avons procédé à des tests $t$ indépendants ainsi que des analyses de covariance (ANCOVA) afin de déterminer les différences statistiquement significatives répondant à nos trois objectifs de recherche. Les ANCOVA ont permis d'associer une partie de la variance des variables à l'intervention et non aux différents prétests. Le design expérimental des quatre groupes de Solomon a été utilisé afin de valider le rendement des élèves lors de chacune des interventions.

La figure 3 présente le design des analyses tel que présenté dans la recherche de McGahee et Tingen (2000) et est suivi d'une brève explication de celui-ci.

Tableau 1. Le design des quatre groupes de Solomon

\begin{tabular}{|c|c|c|c|}
\hline \multicolumn{4}{|c|}{ Intervention sans contrôle inhibiteur } \\
\hline Groupe & $\begin{array}{c}\text { Passation } \\
\text { prétests }\end{array}$ & Intervention & $\begin{array}{l}\text { Passation } \\
\text { posttests }\end{array}$ \\
\hline $\begin{array}{l}1 \\
\text { Intervention } \\
\text { SANS } \\
\text { contrôle } \\
\text { inhibiteur }\end{array}$ & $\mathrm{X}$ & $\mathrm{X}$ & $\mathrm{X}$ \\
\hline $\begin{array}{l}2 \\
\text { Enseignement } \\
\text { régulier }\end{array}$ & $\mathrm{X}$ & & $\mathrm{X}$ \\
\hline $\begin{array}{l}3 \\
\text { Intervention } \\
\text { SANS } \\
\text { contrôle } \\
\text { inhibiteur }\end{array}$ & & $\mathrm{X}$ & $\mathrm{X}$ \\
\hline $\begin{array}{l}4 \\
\text { Enseignement } \\
\text { régulier }\end{array}$ & & & $\mathrm{X}$ \\
\hline \multicolumn{4}{|c|}{ Intervention avec contrôle inhibiteur } \\
\hline $\begin{array}{l}5 \\
\text { Intervention } \\
\text { AVEC } \\
\text { contrôle } \\
\text { inhibiteur }\end{array}$ & $\mathrm{X}$ & $\mathrm{X}$ & $\mathrm{X}$ \\
\hline $\begin{array}{l}6 \\
\text { Enseignement } \\
\text { régulier }\end{array}$ & $\mathrm{X}$ & & $\mathrm{X}$ \\
\hline $\begin{array}{l}7 \\
\text { Intervention } \\
\text { AVEC } \\
\text { contrôle } \\
\text { inhibiteur }\end{array}$ & & $\mathrm{X}$ & $\mathrm{X}$ \\
\hline $\begin{array}{l}8 \\
\text { Enseignement } \\
\text { régulier }\end{array}$ & & & $\mathrm{X}$ \\
\hline
\end{tabular}




\begin{tabular}{|c|c|c|c|}
\hline Groupe & $\begin{array}{c}\text { Passation } \\
\text { prétest }\end{array}$ & Intervention & $\begin{array}{c}\text { Passation } \\
\text { posttest }\end{array}$ \\
\hline & & (1) & \\
\hline 1 & $8)^{x}$ & $\mathbf{X}$ & $\mathbf{x}$ \\
\hline 2 & $x_{x}$ & (2) & $\rightarrow \mathbf{x}$ \\
\hline 3 & & $x$ (7) & $\mathbf{X}$ \\
\hline 4 & & & \begin{tabular}{l|l} 
\\
$x^{6}$
\end{tabular} \\
\hline
\end{tabular}

Figure 3. Design expérimental des quatre groupes de Solomon

Les deux premiers groupes de la conception du design de Solomon sont conçus et interprétés de la même manière que dans la conception prétest-posttest, et fournissent les mêmes contrôles sur la randomisation. La comparaison entre les résultats posttests des groupes 3 et 4, marqués par la ligne « 6 », permet au chercheur de déterminer si l'acte même de prétest influence les résultats. Si l'écart entre les résultats des posttests des groupes 3 et 4 , marqué par la ligne « 6 », diffère de celui des groupes 1 et 2 , marqué par la ligne « 3 », on peut supposer que le prétest a eu un certain effet sur les résultats.

La comparaison entre le prétest du groupe 2 et le posttest du groupe 4 , marquée par la ligne « 7 », permet d'établir si des facteurs externes pourraient avoir provoqué une distorsion temporelle. Cela s'avère une précaution supplémentaire pour s'assurer qu'aucun autre facteur n'ait influencé les résultats au posttest; entres autres l'effet de la passation du prétest comme outil d'apprentissage pour la passation du posttest sans qu'il y ait d'intervention.

La comparaison entre le groupe 1 posttest et le groupe 3 posttest, marquée par la ligne « $4 »$, permet au chercheur de déterminer l'effet que le prétest a eu sur le traitement. Si les résultats posttests pour ces deux groupes diffèrent, alors le prétest a eu un certain effet sur le traitement et l'expérience présente un biais de validité interne.

La comparaison entre le posttest du groupe 2 et le posttest du groupe 4 , marquée par la ligne « 5 », indique si le prétest lui-même a affecté le comportement, indépendamment du traitement. Si les résultats sont significativement différents, alors le prétest a influencé les résultats globaux. Ainsi, ce design d'analyses est utilisé pour l'intervention ciblant l'apprentissage du sens des nombres et du lien entre le sens des nombres et le nombre symbolique, puis pour l'intervention ciblant le sens des nombres, le lien entre ce sens des nombres et le nombre symbolique et le contrôle inhibiteur. En terminant, il y a eu comparaison des deux interventions mentionnées précédemment.
La section suivante présente les différents résultats répondant à nos trois objectifs de recherche.

\section{Résultats}

L'analyse de variance (ANOVA) a permis de dégager qu'il existe une différence statistiquement significative entre les trois types d'interventions (intervention avec deux prérequis, intervention avec trois prérequis et enseignement régulier) pour tous les tests ${ }^{3}$ : le Numeracy Screener non symbolique, $F(2,125)=12,486, p<0,001 ; \eta^{2}=0,169$, le Numeracy Screener symbolique, $F(2,125)=4,036, p=0,020 ; \eta^{2}=0,061$, le TediMath, $F(2,125)=16,575, p<0,001 ; \eta^{2}=0,212$, le test sur le lien entre le nombre symbolique et non symbolique, $F(2,125)=8,060, p<0,001 ; \eta^{2}=0,116$, et les tests nécessitant d'inhiber en contexte numérique. $F(2,125)=37,200, p<0,001$; $\eta^{2}=0,377$. Ces résultats ont permis de poursuivre les analyses.

3.1 Effets d'une intervention ciblant les deux premiers prérequis versus un enseignement régulier

Afin de vérifier si les groupes ayant vécu l'intervention 1 portant sur les deux premiers prérequis (groupes 1 et 3 ) présentent une différence statistiquement significative aux résultats de leur posttest comparativement aux groupes ayant vécu un enseignement régulier (groupes 2 et 4) (objectif $1)$, un test $t$ pour échantillons indépendants a été effectué. Il y a une différence statistiquement significative entre les résultats des groupes ayant vécu l'intervention, $M=24,72$, $E ́ T=8,01$, et ceux des groupes ayant vécu un enseignement régulier, $M=20,33$, ÉT $=8,27$, au test du Numeracy Screener non symbolique, $t(57)=2,066, p=0,043 ; \eta^{2}=0,07$.

Une différence statistiquement significative existe également entre les groupes ayant vécu lintervention, $M=21,03$, $E ́ T=11,32$, et les groupes ayant vécu un enseignement régulier, $M=13,44$, ÉT $=7,82$, au test du Numeracy Screener symbolique, $t(57)=2,939, p=0,005 ; \eta^{2}=0,13$.

Il y a une différence statistiquement significative entre les groupes ayant vécu l'intervention, $M=2.53$, ÉT $=0,57$, et les groupes ayant vécu un enseignement régulier, $M=2,07$, ÉT $=0,36$, au test Tedi-Math, $t(57)=3,622, p=0,001 ; \eta^{2}=0,19$.

Également, une différence statistiquement significative existe entre les groupes ayant vécu lintervention, $M=0,82$, É $T=0,23$, et les groupes ayant vécu l'enseignement régulier, $M=0,66$, ÉT $=0,18$, au test sur le lien entre le nombre symbolique et non symbolique, $t(57)=-2,801, p=0,007 ; \eta^{2}=0,12$.

Finalement, une différence statistiquement significative existe entre les groupes ayant vécu l'intervention, $M=5,44$, $E ́ T=2,22$, et les groupes ayant vécu l'enseignement régulier, $M=2,25$, ÉT $=1,96$, aux tests nécessitant d'inhiber en contexte numérique, $t(57)=5,791, p<0,001 ; \eta^{2}=0,37$.

\footnotetext{
${ }^{3}$ Le seuil significatif de l'homogénéité des variances est fixé à $p<0,05$ et le seuil significatif de la normalité est fixé à $p>0,05$.
} 
Puisque les résultats des analyses se sont avérés statistiquement significatifs, une analyse de covariance a été effectuée afin d'évaluer l'effet même de l'intervention après avoir contrôlé l'effet des prétests. Les conditions de normalité, d'homogénéité et d'interaction sont satisfaites. Les résultats du tableau 2 démontrent l'effet de l'intervention sur certains prérequis.

Tableau 2. Résultats de l'ANCOVA comparant les groupes ayant vécu une intervention ciblant le sens des nombres et le lien entre ce sens des nombres et le nombre symbolique (groupe 1) versus ceux ayant vécu un enseignement régulier (groupe 2)

\begin{tabular}{llll}
\hline Test & & $F$ & Éta-carré \\
\hline \multirow{2}{*}{$\begin{array}{l}\text { Numeracy Screener non } \\
\text { symbolique }\end{array}$} & Intervention & $F(1,30)=5,682, p=0,024$ & $\eta^{2}=0,159$ \\
\cline { 2 - 4 } & Prétest & $F(1,30)=5,271, p=0,029$ & $\eta^{2}=0,149$ \\
\hline Numeracy Screener symbolique & Intervention & $F(1,30)=1,258, p=0,271$ & $\eta^{2}=0,328$ \\
\cline { 2 - 4 } & Prétest & $F(1,30)=14,627, p=0,001$ & $\eta^{2}=0,626$ \\
\hline Tedi-Math & Intervention & $F(1,30)=2,132, p=0,155$ & \\
\cline { 2 - 4 } & Prétest & $F(1,30)=50,179, p<0,001$ & $\eta^{2}=0,169$ \\
\hline $\begin{array}{l}\text { Test sur le lien entre le nombre } \\
\text { symbolique et non symbolique }\end{array}$ & Intervention & $\mathrm{F}(1,30)=0,083, p=0,776$ & $\eta^{2}=0,236$ \\
\cline { 2 - 4 } $\begin{array}{l}\text { Test nécessitant d'inhiber en } \\
\text { contexte numérique }\end{array}$ & Intervention & $F(1,30)=6,320, p=0,017$ & $\eta^{2}=0,474$ \\
\cline { 2 - 4 }
\end{tabular}

Comme le démontrent les résultats, la taille de l'effet de l'intervention au Numeracy Screener non symbolique et aux tests nécessitant d'inhiber en contexte numérique, telle qu'évaluée par le $\eta^{2}$, est grande (Cohen, 1988). L'intervention explique $16 \%$ de la variance des données obtenues avec le Numeracy Screener non symbolique et $24 \%$ de la variance des données obtenues par le test nécessitant d'inhiber en contexte numérique. Ces résultats sont repris dans la section Discussion.

3.2 Effets d'une intervention ciblant les deux premiers prérequis ainsi que le contrôle inhibiteur versus un enseignement régulier

Un test $t$ pour échantillons indépendants a été effectué afin de vérifier si les groupes ayant vécu l'intervention sur les deux premiers prérequis et le contrôle inhibiteur (groupes 5 et 7) présentent une différence statistiquement significative aux résultats de leur posttest comparativement aux groupes ayant vécu un enseignement régulier (groupes 6 et 8 ) (objectif 2). Il y a une différence statistiquement significative entre les scores de performance des groupes ayant vécu l'intervention ciblant les deux premiers prérequis et le contrôle inhibiteur, $M=26,09$, ÉT $=8,25$, et les scores de performance de ceux ayant vécu un enseignement régulier, $M=15,91, \quad$ ÉT $=9,05$ au test Numeracy Screener non symbolique, $t(65)=4,816, p<0,001 ; \eta^{2}=0,26$.

Il existe une différence statistiquement significative entre les scores de performance des groupes ayant vécu l'intervention ciblant les deux prérequis et le contrôle inhibiteur, $M=2,74$, ÉT $=0.43$, et les scores de performance de ceux qui ont vécu un enseignement régulier, $M=2,21$, ÉT $=0,59$, au test TediMath, $\mathrm{t}(65)=4,210, p<0,001 ; \eta^{2}=0,21$.
Il existe une différence statistiquement significative entre les scores de performance des groupes ayant vécu l'intervention ciblant les deux prérequis et le contrôle inhibiteur, $M=0,77$, ÉT $=0,18$, et les scores de performance de ceux ayant reçu un enseignement régulier, $M=0,65$, ÉT $=0,19$ au test sur le lien entre le nombre symbolique et non symbolique, $t(65)=2,638$, $p=0,010 ; \eta^{2}=0,10$.

Finalement, il existe une différence statistiquement significative entre les scores de performance des groupes ayant vécu l'intervention ciblant deux prérequis et le contrôle inhibiteur, $M=6,23$, ÉT $=2,21$, et les scores de performance de ceux ayant vécu un enseignement régulier, $M=2.66$, ÉT $=2,44$, au test nécessitant d'inhiber en contexte numérique, $t(65)=6.280, p<0,001 ; \eta^{2}=0,38$.

Cependant, il n'y a pas de différence statistiquement significative entre les scores de performance des groupes ayant vécu cette même intervention, $M=20,09$, ÉT $=8,83$, et les scores de performance de ceux ayant vécu un enseignement régulier, $M=17,67$, ÉT $=9,29$, bien que les scores moyens de ceux ayant vécu l'intervention soient plus élevés que ceux des groupes ayant vécu l'enseignement régulier au test du Numeracy Screener symbolique, $t(65)=1,094, p=0.278$.

Suivant ces analyses, une analyse de covariance a été effectuée afin d'évaluer l'effet même de l'intervention après avoir contrôlé l'effet des prétests pour chacun des tests à l'étude. Les conditions de normalité, d'homogénéité et d'interaction sont satisfaites. Les résultats du tableau 3 montrent une différence significative au niveau de la plupart des tests. 
Tableau 3. Résultats de l'ANCOVA comparant les groupes ayant vécu une intervention ciblant le sens des nombres, le lien entre ce sens des nombres et le nombre symbolique, et le contrôle inhibiteur (groupe 5) versus ceux ayant vécu un enseignement régulier (groupe 6)

\begin{tabular}{|c|c|c|c|}
\hline Test & & $\mathrm{F}$ & Éta-carré \\
\hline \multirow{2}{*}{$\begin{array}{l}\text { Numeracy Screener non } \\
\text { symbolique }\end{array}$} & Intervention & $F(1,29)=11,082, p=0,002$ & $\eta^{2}=0,276$ \\
\hline & Prétest & $F(1,29)=7,468, p=0,011$ & $\eta^{2}=0,205$ \\
\hline \multirow[t]{2}{*}{ Numeracy Screener symbolique } & Intervention & $\mathrm{F}(1,29)=2,585, p=0,119$ & \\
\hline & Prétest & $F(1,29)=5,788, p=0,023$ & $\eta^{2}=0,166$ \\
\hline \multirow[t]{2}{*}{ Tedi-Math } & Intervention & $F(1,29)=18,810, p<0,001$ & $\eta^{2}=0,393$ \\
\hline & Prétest & $F(1,29)=40,933, p<0,001$ & $\eta^{2}=0,585$ \\
\hline \multirow{2}{*}{$\begin{array}{l}\text { Test sur le lien entre le nombre } \\
\text { symbolique et non symbolique }\end{array}$} & Intervention & $\mathrm{F}(1,29)=10,660, p=0,003$ & $\eta^{2}=0,269$ \\
\hline & Prétest & $F(1,29)=15,844, p<0,001$ & $\eta^{2}=0,353$ \\
\hline \multirow{2}{*}{$\begin{array}{l}\text { Test nécessitant d'inhiber en } \\
\text { contexte numérique }\end{array}$} & Intervention & $F(1,29)=32,412, p<0,001$ & $\eta^{2}=0,528$ \\
\hline & Prétest & $F(1,29)=14,359, p=0,001$ & $\eta^{2}=0,331$ \\
\hline
\end{tabular}

Comme le démontrent les résultats, la taille de l'effet de l'intervention au test Numeracy Screener non symbolique, au Tedi-Math, au test sur le lien entre le nombre symbolique et non symbolique et au test nécessitant d'inhiber en contexte numérique, telle qu'évaluée par le $\eta^{2}$, est grande. L'intervention explique $28 \%$ de la variance des scores du Numeracy Screener non symbolique, 39 \% des scores du TediMath et $27 \%$ des scores du test du lien entre le nombre symbolique et non symbolique. Finalement, l'intervention explique $53 \%$ des scores du test nécessitant d'inhiber en contexte numérique.

3.3 Effets d'une intervention ciblant les deux premiers prérequis ainsi que le contrôle inhibiteur versus une intervention ciblant seulement les deux premiers prérequis

Afin d'atteindre notre troisième objectif de recherche, soit comparer l'efficacité de l'intervention visant l'acquisition des deux prérequis mathématiques (groupes 1 et 3 ) à une intervention visant l'acquisition de ces deux mêmes prérequis ainsi que le contrôle inhibiteur (groupes 5 et 7), un test $t$ pour échantillons indépendants a été effectué.

Le tableau 4 montre que, bien que la plupart des moyennes des groupes ayant vécu l'intervention avec contrôle inhibiteur soient plus élevées que celles de l'intervention sans contrôle inhibiteur, l'écart ne fut pas suffisant pour démontrer un effet statistiquement significatif.
Par contre, puisque les résultats des différentes analyses en lien avec les deux types d'intervention et l'enseignement régulier étaient différents et que l'intervention avec contrôle inhibiteur a eu un effet sur différents tests versus l'intervention sans contrôle inhibiteur (sur le test du TediMath et sur le test du lien entre le nombre symbolique et non symbolique), nous avons décidé de refaire des analyses statistiques en analysant tous les sous-tests du Tedi-Math (les épreuves d'opérations logiques, liées au système numérique, au dénombrement et au comptage furent analysées) et ceux du test sur les énoncés contre-intuitifs.

Ainsi, un test $t$ pour échantillons indépendants s'est avéré statistiquement significatif pour le sous-test comptage faisant partie du Tedi-Math, $t(64)=3,050, p 0,03 ; \eta^{2}=0,13$ ) et le sous-test de la conservation du nombre faisant partie du test nécessitant d'inhiber en contexte numérique, $t(64)=2,438, p=0,018 ; \eta^{2}=0,08$. L'ensemble des résultats est présenté dans le tableau 5 .

À la lumière de ces résultats, nous pouvons constater que l'intervention avec contrôle inhibiteur, en plus de mener à des scores de performance supérieurs sur l'ensemble des prérequis à l'étude par rapport à un groupe ayant vécu un enseignement régulier, tel que vu précédemment, mène à des scores de performance plus élevés pour l'habileté de comptage et pour l'habileté à conserver le nombre, comparativement à l'intervention sans contrôle inhibiteur. 
Tableau 4. Résultats obtenus à chacun des tests pour les groupes ayant vécu une intervention (avec et sans contrôle inhibiteur)

\begin{tabular}{|c|c|c|c|c|c|}
\hline Test & Type intervention & $\mathrm{N}$ & M & ÉT & Test $t$ \\
\hline \multirow[t]{2}{*}{ Numeracy Screener non symbolique } & $\begin{array}{l}\text { AVEC contrôle } \\
\text { inhibiteur }\end{array}$ & 34 & 26,09 & 8,25 & \multirow[t]{2}{*}{$\begin{array}{l}t(64)=0,684 \\
p=0,496\end{array}$} \\
\hline & $\begin{array}{l}\text { SANS contrôle } \\
\text { inhibiteur }\end{array}$ & 32 & 24,72 & 8,00 & \\
\hline \multirow[t]{2}{*}{ Numeracy Screener symbolique } & $\begin{array}{l}\text { AVEC contrôle } \\
\text { inhibiteur }\end{array}$ & 34 & 20,09 & 8,83 & \multirow[t]{2}{*}{$\begin{array}{l}t(64)=-0,379 \\
p=0,706\end{array}$} \\
\hline & $\begin{array}{l}\text { SANS contrôle } \\
\text { inhibiteur }\end{array}$ & 32 & 21,03 & 11,32 & \\
\hline \multirow[t]{2}{*}{ Tedi-Math } & $\begin{array}{l}\text { AVEC contrôle } \\
\text { inhibiteur }\end{array}$ & 34 & 2,74 & 0,434 & \multirow[t]{2}{*}{$\begin{array}{l}t(64)=1,762 \\
p=0,083\end{array}$} \\
\hline & $\begin{array}{l}\text { SANS contrôle } \\
\text { inhibiteur }\end{array}$ & 32 & 2,53 & 0,57 & \\
\hline \multirow[t]{2}{*}{$\begin{array}{l}\text { Test sur le lien entre le nombre } \\
\text { symbolique et non symbolique }\end{array}$} & $\begin{array}{l}\text { AVEC contrôle } \\
\text { inhibiteur }\end{array}$ & 34 & 0,82 & 0,18 & \multirow[t]{2}{*}{$\begin{array}{l}t(64)=-0,924 \\
p=0,359\end{array}$} \\
\hline & $\begin{array}{l}\text { SANS contrôle } \\
\text { inhibiteur }\end{array}$ & 32 & 0,82 & 0,23 & \\
\hline \multirow[t]{2}{*}{$\begin{array}{l}\text { Test nécessitant d'inhiber en } \\
\text { contexte numérique }\end{array}$} & $\begin{array}{l}\text { AVEC contrôle } \\
\text { inhibiteur }\end{array}$ & 34 & 6,23 & 2,21 & \multirow[t]{2}{*}{$\begin{array}{l}t(64)=1,443 \\
p=0,154\end{array}$} \\
\hline & $\begin{array}{l}\text { SANS contrôle } \\
\text { inhibiteur }\end{array}$ & 32 & 5,44 & 2,22 & \\
\hline
\end{tabular}

Tableau 5. Différences de moyennes, aux posttests, entre les groupes ayant vécu une intervention sur les deux prérequis avec contrôle inhibiteur et ceux ayant vécu une intervention sur les deux prérequis sans contrôle inhibiteur, incluant les sous-tests du Tedi-Math et le test sur les énoncés contre-intuitifs

\begin{tabular}{|c|c|c|c|c|c|}
\hline Test & Intervention & M & ÉT & Test $t$ & Taille d'effet $\eta^{2}$ \\
\hline \multirow{2}{*}{$\begin{array}{l}\text { Test nécessitant d'inhiber en } \\
\text { contexte numérique } \\
\text { (petits points versus gros points) }\end{array}$} & $\begin{array}{l}\text { AVEC contrôle } \\
\text { inhibiteur }\end{array}$ & 8,06 & 3,61 & \multirow[t]{2}{*}{$\begin{array}{l}t(64)=-0,878 \\
p=0,383\end{array}$} & \\
\hline & $\begin{array}{l}\text { SANS contrôle } \\
\text { inhibiteur }\end{array}$ & 7,31 & 3,28 & & \\
\hline \multirow{2}{*}{$\begin{array}{l}\text { Test nécessitant d'inhiber en } \\
\text { contexte numérique } \\
\text { (petits chiffres versus gros chiffres) }\end{array}$} & $\begin{array}{l}\text { AVEC contrôle } \\
\text { inhibiteur }\end{array}$ & 7,91 & 3,76 & \multirow[t]{2}{*}{$\begin{array}{l}t(64)=0,874 \\
p=0,385\end{array}$} & \\
\hline & $\begin{array}{l}\text { SANS contrôle } \\
\text { inhibiteur }\end{array}$ & 7,13 & 7,13 & & \\
\hline \multirow{2}{*}{$\begin{array}{l}\text { Test nécessitant d'inhiber en } \\
\text { contexte numérique } \\
\text { (conservation du nombre) }\end{array}$} & $\begin{array}{l}\text { AVEC contrôle } \\
\text { inhibiteur }\end{array}$ & 2,71 & 1,38 & \multirow[t]{2}{*}{$\begin{array}{l}t(64)=-2,438 \\
p=0,018\end{array}$} & $\eta^{2}=0,08$ \\
\hline & $\begin{array}{l}\text { SANS contrôle } \\
\text { inhibiteur }\end{array}$ & 1,88 & 1,39 & & \\
\hline \multirow[t]{2}{*}{$\begin{array}{l}\text { Tedi-Math } \\
\text { (opérations logiques) }\end{array}$} & $\begin{array}{l}\text { AVEC contrôle } \\
\text { inhibiteur }\end{array}$ & 1,29 & 0,87 & \multirow[t]{2}{*}{$\begin{array}{l}t(64)=-0,405 \\
p=0,687\end{array}$} & \\
\hline & $\begin{array}{l}\text { SANS contrôle } \\
\text { inhibiteur }\end{array}$ & 1,21 & 1,31 & & \\
\hline \multirow[t]{2}{*}{$\begin{array}{l}\text { Tedi-Math } \\
\text { (système numérique) }\end{array}$} & $\begin{array}{l}\text { AVEC contrôle } \\
\text { inhibiteur }\end{array}$ & 7,14 & 0,87 & \multirow[t]{2}{*}{$\begin{array}{l}t(64)=0,285 \\
p=0,084\end{array}$} & \\
\hline & $\begin{array}{l}\text { SANS contrôle } \\
\text { inhibiteur }\end{array}$ & 6,84 & 1,31 & & \\
\hline \multirow[t]{2}{*}{$\begin{array}{l}\text { Tedi-Math } \\
\text { (dénombrement) }\end{array}$} & $\begin{array}{l}\text { AVEC contrôle } \\
\text { inhibiteur }\end{array}$ & 1,55 & 0,24 & \multirow[t]{2}{*}{$\begin{array}{l}t(50,768)=1,789 \\
p=0,084\end{array}$} & \\
\hline & $\begin{array}{l}\text { SANS contrôle } \\
\text { inhibiteur }\end{array}$ & 1,42 & 0,38 & & \\
\hline \multirow[t]{2}{*}{$\begin{array}{l}\text { Tedi-Math } \\
\text { (comptage) }\end{array}$} & $\begin{array}{l}\text { AVEC contrôle } \\
\text { inhibiteur }\end{array}$ & 0,99 & 0,53 & \multirow[t]{2}{*}{$\begin{array}{l}t(64)=-3,050 \\
p=0,03\end{array}$} & \multirow[t]{2}{*}{$\eta^{2}=0,13$} \\
\hline & $\begin{array}{l}\text { SANS contrôle } \\
\text { inhibiteur }\end{array}$ & 0,64 & 0,40 & & \\
\hline
\end{tabular}




\section{Discussion}

Les différentes analyses réalisées ont permis de répondre aux trois objectifs de recherche, soit de :

1. comparer l'efficacité d'une intervention, au préscolaire, visant l'acquisition de deux prérequis mathématiques : le sens des nombres et le lien entre le sens des nombres et le nombre symbolique, à un enseignement régulier des mathématiques;

2. comparer l'efficacité d'une intervention, au préscolaire, visant l'acquisition de deux prérequis mathématiques : le sens des nombres, le lien entre le sens des nombres et le nombre symbolique, ainsi que le contrôle inhibiteur, à un enseignement régulier des mathématiques;

3. comparer l'efficacité d'une intervention, au préscolaire, visant l'acquisition de deux prérequis mathématiques : le sens des nombres et le lien entre le sens des nombres et le nombre symbolique, à une intervention visant plutôt l'acquisition de deux prérequis mathématiques : le sens des nombres, le lien entre le sens des nombres et le nombre symbolique, ainsi que le contrôle inhibiteur.

Dans un premier temps, il sera question de l'effet des deux interventions (sans et avec contrôle inhibiteur) comparativement à un enseignement régulier. Dans un second temps, il sera question de l'effet de l'intervention avec contrôle inhibiteur comparativement à l'effet de l'intervention sans contrôle inhibiteur.

4.1 Effets des deux interventions (sans et avec contrôle inhibiteur) comparativement à un enseignement régulier

En examinant l'analyse des différents tests t présentés, nous pouvons constater que les deux types d'intervention ont eu un effet significatif comparativement à un enseignement régulier (objectifs 1 et 2).

Les résultats des différents tests pour les deux types d'intervention (sans et avec contrôle inhibiteur) comparativement à un enseignement régulier ont mené à des scores de performance plus élevés pour l'ensemble des tests mesurant les prérequis à l'étude, à l'exception du test Numeracy Screener symbolique. Cette exception est difficile à expliquer, mais elle est peut-être, au moins en partie, causée par le fait que les habiletés de comparaison de nombres symboliques mesurées par le Numeracy Screener symbolique sont celles qui sont le plus explicitement développées chez les élèves du groupe ayant reçu un enseignement régulier basé sur le PFÉQ; il y aurait donc moins d'écart entre les deux groupes pour ce type d'habiletés. Toutefois, cette différence amène un questionnement quant aux activités présentes dans l'intervention. Bien que celles-ci soient nombreuses, il serait possiblement intéressant d'intégrer le logiciel Number Race (Wilson et al., 2006) à lintervention. Cet ajout permettrait de travailler la comparaison des nombres symboliques faisant partie du prérequis du lien entre le sens des nombres et le nombre symbolique. Lors de l'expérimentation du logiciel, les élèves ont présenté une augmentation spécifique de leur performance sur les tâches en lien avec le sens des nombres. La vitesse de la capacité à subitiser et l'habileté liée à la comparaison numérique ont augmenté de plusieurs centaines de millisecondes. Ainsi, l'ajout de temps de jeu consacré à ce logiciel aiderait fort probablement les élèves à développer de façon significative la comparaison de nombres symboliques et ainsi, le deuxième prérequis.

Ensuite, les résultats de l'analyse de covariance ont permis d'observer une augmentation considérable des scores de performance des élèves des groupes ayant vécu l'intervention (avec et sans contrôle inhibiteur) comparativement aux groupes de contrôle, et ce, pour l'ensemble des tests. En contrepartie, une fois l'effet du prétest contrôlé, les résultats nous indiquent que les deux types d'intervention mènent à des scores de performance supérieurs quant au prérequis du sens du nombre (test du Numeracy Screener non symbolique) et quant au prérequis de la capacité d'inhiber (tests nécessitant d'inhiber en contexte numérique).

Cependant, seule l'intervention avec contrôle inhibiteur, une fois l'effet du prétest contrôlé, a permis d'associer une partie de la variance à la variable du prérequis lié au lien entre le sens des nombres et le nombre symbolique (test Tedi-Math et test du lien entre le nombre symbolique et non symbolique).

En résumé, les résultats associés aux deux premiers objectifs de notre recherche ont permis de constater l'impact positif sur les habiletés en mathématique des deux interventions comparativement à un enseignement régulier. Cependant, seule l'intervention avec contrôle inhibiteur, une fois l'effet du prétest contrôlé, a mené à des scores de performance supérieurs pour les trois prérequis; ce qu'il n'a pas été possible de démontrer par l'intervention sans contrôle inhibiteur.

En somme, l'intervention avec contrôle inhibiteur, en plus d'avoir un impact sur l'acquisition du sens des nombres et du contrôle inhibiteur, permet également, contrairement à l'intervention sans contrôle inhibiteur, d'avoir un impact sur le lien entre le sens des nombres et le nombre symbolique comparativement à l'enseignement régulier.

\subsection{Effets de l'intervention avec contrôle inhibiteur versus sans contrôle inhibiteur}

Pour répondre au troisième objectif de la recherche, nous avons comparé l'impact sur les habiletés en mathématique d'une intervention ciblant les deux premiers prérequis et le contrôle inhibiteur à une intervention ciblant seulement les deux premiers prérequis. Ce qui ressort des analyses statistiques réalisées est les différences au niveau de que la partie comptage du test Tedi-Math, et du test de la conservation du nombre réalisé par l'équipe de recherche se sont avérées statistiquement significatives. Ces résultats nous indiquent qu'un enseignement incluant le contrôle inhibiteur favoriserait davantage l'acquisition du comptage et de la conservation du nombre contrairement à un enseignement sans contrôle inhibiteur. 
En ce sens, la recherche d'Imbert (2005) démontre un lien entre l'habileté de comptage et le contrôle inhibiteur. Comme le propose également Baroody (Baroody, 1991; Baroody et Price, 1983), il semble que l'interaction entre les habiletés liées à la chaine numérique d'une part, et les concepts de comptage d'autre part, soit à l'origine de l'acquisition de la procédure de comptage. Selon la tâche à effectuer, les enfants activeraient soit des schémas fondés sur les connaissances conceptuelles (schéma efficace), soit des schémas fondés sur la recherche de la réponse par une utilisation et une manipulation de la chaine numérique, c'està-dire par des procédures (schéma non efficace). Ainsi, la recherche d'Imbert (2005) a démontré l'existence de différences par rapport aux ressources cognitives entre les différents profils d'acquisition (connaissances conceptuelles ou procédurales). En effet, les résultats de cette recherche ont démontré que les enfants faisant partie du groupe ayant des difficultés d'apprentissage obtenaient globalement des performances plus faibles que les autres enfants aux épreuves de mémoire, de contrôle inhibiteur et de lexique. Ces conclusions vont dans le même sens que les recherches de Bernoussi (2002), Geary, Hamson et Hoard (2000), Klein et Bisanz (2000), et Lépine, Camos et Barrouillet (2003).

Ainsi, selon les recherches d'Imbert (2005) et de Camos (2003), le fait que l'intervention avec contrôle inhibiteur puisse mener à des scores de performance supérieurs au sous-test comptage du test Tedi-Math n'est pas une surprise en soi, puisque leurs recherches ont permis de mettre en évidence que le développement du dénombrement résulterait d'un changement de stratégies concernant la diminution du cout cognitif de la stratégie primitive de dénombrement «un par un» (connaissances procédurales) et dans le rôle majeur des ressources cognitives, entres autres le contrôle inhibiteur. Ainsi, ces résultats confirmeraient l'intérêt d'une intervention par contrôle inhibiteur pour développer le lien entre le sens des nombres et le nombre symbolique.

En contrepartie, l'intervention par contrôle inhibiteur semble également être nécessaire pour favoriser la capacité de conserver le nombre. Pour conserver l'apparence du nombre, les élèves doivent non seulement inhiber leur fausse conception que la longueur de la distribution influence le nombre, mais également dénombrer et comparer les deux quantités. Ainsi, l'enseignement par contrôle inhibiteur permettrait aux élèves de contrer les pièges en lien avec la conservation du nombre. Ceci va dans le même sens que la recherche d'Houdé et al. (2011) sur la conservation du nombre.

Malgré ces deux résultats précédents significatifs, des résultats non significatifs ont été obtenus en lien avec les deux autres tâches nécessitant d'inhiber en contexte numérique, soit le test sur la comparaison des ensembles de petits et gros points et le test sur la comparaison des petits et gros chiffres, ce qui peut surprendre étant donné la littérature scientifique existante à ce sujet.

La prochaine section aborde ces deux tests dont la différence des scores s'est révélée statistiquement non significative entre les groupes ayant vécu l'intervention avec contrôle inhibiteur et les groupes ayant vécu l'intervention sans contrôle inhibiteur.

4.2.1 Test sur la comparaison des ensembles de petits et gros points

Comme mentionné dans la recherche de Dehaene (2011), le sens des nombres serait présent dès les premiers mois de vie dans le cerveau des enfants et leur permettrait d'avoir une idée de la grandeur des nombres, ainsi qu'une compréhension du nombre sous sa forme non symbolique. Le test sur la comparaison des ensembles de petits et gros points permettait aux élèves de comparer deux ensembles de points non symboliques variant au niveau de la taille des points présentés. Ce test vérifie l'acquisition du sens des nombres chez les élèves. Puisque, tel que démontré par les recherches de Dehaene (2011), le sens des nombres est perçu comme le sens de l'approximation des nombres et serait la base de la construction des compétences en mathématiques, il serait présent chez les élèves lors de leur entrée au préscolaire. De plus, tel que mentionné dans le programme d'éducation préscolaire québécois (MELS, 2013), seul ou avec un peu d'aide, l'élève de cet âge doit faire correspondre, à l'oral, un nombre inférieur à cinq à la quantité d'objets correspondante et il doit reconnaitre la différence entre beaucoup et peu. Ces connaissances sont en lien avec la tâche de comparaison des petits et gros points. En ce sens, nous croyons qu'il y aurait probablement eu un enseignement de comparaison d'ensembles non symboliques chez ces élèves avant la passation du test, ce qu'il aurait fallu vérifier auprès de l'enseignante avant la passation.

\subsubsection{Test sur la comparaison de petits et gros chiffres}

Le nombre sous sa forme symbolique se construit à partir du sens des nombres. Puis, au cours de son apprentissage du langage oral, l'élève acquiert progressivement les nombres sous leur forme symbolique. Cette acquisition nécessite du temps et l'acquisition de plusieurs concepts (Fayol, 2008, 2012; Fuson, 1991). Pour ces raisons, l'acquisition du système symbolique chez l'élève de 5 ans est quelque chose de très complexe. De plus, en comparant avec les résultats obtenus lors des différentes analyses statistiques, il semble que l'élève de cet âge (5 ans), au mois d'octobre, reconnaisse le code écrit; soit les chiffres, mais non les nombres issus des chiffres (cardinalité). Il est possible que les élèves de tous les groupes aient eu tendance à choisir tous les nombres à droite lors des comparaisons, puisque selon l'ordre des nombres, le nombre le plus élevé se situe à droite du précédent. Ainsi, le symbolisme n'est pas perçu comme le représentant du nombre, mais comme un chiffre; un ordre de grandeur.

De plus, tel que vu précédemment, les habiletés liées au comptage aident en grande partie à construire le nombre symbolique. En fait, le nombre sous sa forme symbolique prend appui sur le sens des nombres à partir de la même zone cérébrale, soit les sillons intrapariétaux (Dehaene, 2011). Puis, par le principe de recyclage neuronal, la zone associée au sens des nombres se reconvertirait pour accueillir le nombre sous sa forme symbolique. Cette reconversion est possible 
par le type d'activités proposées qui mettent de l'avant le concept de nombre.

Les élèves de 5 ans participant à cette étude n'ont peut-être pas eu assez d'occasions d'être mis en relation avec les nombres symboliques et d'y associer toutes les composantes liées au nombre et non seulement au code écrit, soit le chiffre. C'est probablement pour cette raison que l'enseignement par contrôle inhibiteur n'a pas eu un effet statistiquement significatif comparativement au groupe ayant vécu l'intervention sans contrôle inhibiteur. Il serait toutefois intéressant d'ajouter des activités de relation entre le nombre non symbolique et symbolique dans l'intervention et la faire vivre plus tard dans l'année scolaire. Ainsi, nous pourrions peut-être observer un apport d'un enseignement par contrôle inhibiteur lors de tâche de comparaison de nombres de différentes grandeurs.

En somme, l'ensemble de ces résultats indique qu'un enseignement explicite des trois prérequis en mathématiques (sens des nombres, lien entre le sens des nombres et le nombre symbolique et le contrôle inhibiteur) accentue l'apprentissage de certaines habiletés de base en mathématiques non seulement comparativement à un enseignement régulier, mais également en comparaison avec une intervention sans contrôle inhibiteur.

\subsection{Limites}

Quoique cette recherche ait démontré des résultats positifs et qu'elle enrichisse les connaissances sur le lien entre la didactique des mathématiques et les neurosciences, elle comporte toutefois quelques limites.

Sur le plan méthodologique, certains tests utilisés ne sont pas validés, puisque ce sont des tests élaborés par notre équipe de recherche (tests sur le lien entre le nombre symbolique et non symbolique et tests sur les énoncés contre-intuitifs). Une future recherche pourrait permettre de valider la scientificité de ceux-ci.

De plus, puisque nous savons à présent que l'enseignement du contrôle inhibiteur permet un apport supplémentaire au niveau de l'acquisition du comptage et de la conservation du nombre, il serait pertinent de reconduire une recherche similaire, mais en comparant uniquement les groupes ayant vécu l'intervention sans et avec contrôle inhibiteur. Pour ce faire, il serait pertinent de remplacer les quatre groupes ayant reçu l'enseignement régulier par deux groupes vivant l'intervention sans contrôle inhibiteur, et deux autres l'intervention avec contrôle inhibiteur.

Également, dans le cadre de notre étude, la population ciblée est une population provenant d'un milieu homogène ayant le même statut socioéconomique. Il serait intéressant de faire revivre cette intervention, mais cette fois-ci, en diversifiant le milieu (milieu urbain, rural). Ceci permettrait une représentativité plus grande du contexte social actuel.
De plus, nous avons fait vivre lintervention au mois d'octobre sur une période de cinq semaines. Selon les commentaires reçus des enseignants, il serait mieux de faire vivre l'intervention un peu plus tard dans l'année et de l'échelonner sur un plus grand nombre de semaines. Ceci permettrait aux élèves de passer plus de temps sur certaines activités. Cela permettrait également aux enseignants une meilleure gestion; puisque les activités étaient vécues pour la première fois par les élèves, cela nécessitait beaucoup d'accompagnement de la part de l'enseignant.

\section{Conclusion}

En résumé, bien que l'intervention ciblant le sens des nombres et le lien entre le sens des nombres et le nombre symbolique permette aux élèves de développer ces deux prérequis, lintervention en lien avec ces deux prérequis et le contrôle inhibiteur semble comporter certains avantages supplémentaires, notamment un plus grand développement des habiletés de comptage et de conservation du nombre. Par rapport à un enseignement régulier, les deux interventions de l'étude permettent de développer davantage toutes les habiletés mathématiques de base mesurées par les tests utilisés, à l'exception de la comparaison de nombres symboliques pour le groupe avec contrôle inhibiteur.

Puisque cette recherche a permis de faire ressortir limportance d'un enseignement explicite du contrôle inhibiteur en plus des deux autres prérequis (sens des nombres et le lien entre le sens des nombres et le nombre symbolique), il serait intéressant de reproduire cette recherche, mais de se concentrer uniquement sur le troisième objectif de celle-ci. Cette nouvelle étude permettrait de mieux documenter l'impact de l'enseignement du contrôle inhibiteur dans une intervention mathématique au préscolaire et possiblement d'ouvrir sur le primaire, puisque cet enseignement semble être une voie prometteuse pour l'enseignement des mathématiques. Il faudrait toutefois y considérer les limites énoncées plus haut.

\section{Références}

Ansari, D. (2008). Effects of development and enculturation on number representation in the brain. Nature Reviews. Neuroscience, 9(4), 278-291. https://doi.org/10.1038/nrn2334

Ansari, D., Dhital, B. et Siong, S. C. (2006). Parametric effects of numerical distance on the intraparietal sulcus during passive viewing of rapid numerosity changes. Brain Research, 1067(1), 181-188. https://doi.org/10.1016/j.brainres.2005.10.083

Baroody, A. J. (1991). Procédures et principes de comptage : leur développement avant l'école. Dans J. Bideaud, C. Meljac et J.-P. Fischer (dir.), Les chemins du nombre (p. 133-158). Lille, France : Presses universitaires de Lille. 
Baroody, A. J., Eiland, M. D., Purpura, D. J. et Reid, E. E. (2012). Fostering at-risk kindergarten children's number sense. Cognition and Instruction, 30(4), 435-470. https://doi.org/10.1080/07370008.2012.720152

Baroody, A. J., Eiland, M. et Thompson, B. (2009). Fostering atrisk preschoolers' number sense. Early Education $\varepsilon$ Development, 20(1), 80-128. https://doi.org/10.1080/10409280802206619

Baroody, A. J. et Price, J. (1983). The development of the number-word sequence in the counting of three-yearolds. Journal for Research in Mathematics Education, 14(5), 361-368. https://doi.org/10.2307/748681

Baruk S. (2003). Comptes pour petits et grands. Volume 2: Pour un apprentissage des opérations, des calculs, et des problèmes, fondé sur le langage et le sens. Paris, France: Magnard.

Bégin, G. et Ladouceur, R. (1980). Protocoles de recherche en sciences appliquées et fondamentales. St-Hyacinthe, QC : Edisem.

Bernoussi, M. (2002). Contraintes mnésiques et développementales dans la connaissance des faits arithmétiques. Dans J. Bideaud et H. Le Halle (dir.), Le développement des activités numériques chez l'enfant (p. 175-194). Paris, France : Hermès.

Bideaud, J. et Lehalle, H. (dir.). (2002). Le développement des activités numériques chez l'enfant. Paris, France : Hermès.

Camos, V. (2003). Coordination process in counting. International Journal of Psychology, 38(1), 24-36. https://doi.org/10.1080/00207590244000269

Charron, C., Duquesne, F., Marchand, M.-H. et Meljac, C. (2001). L'évaluation des conduites numériques des enfants en grande difficulté. Dans A. Van Hout et C. Meljac (dir.), Les troubles du calcul et dyscalculies chez l'enfant (p. 336-346). Paris, France : Masson.

Clark, C. A. C., Pritchard, V. E. et Woodward, L. J. (2010). Preschool executive functioning abilities predict early mathematics achievement. Developmental Psychology, 46(5), 1176-1191. https://doi.org/10.1037/a0019672

Clements, D. H. (2007). Curriculum research: Toward a framework for "research-based curricula." Journal for Research in Mathematics Education, 38(1), 35-70.

Clements, D. H., DiBiase, A.-M. et Sarama, J. (dir.). (2004). Engaging young children in mathematics: Standards for early childhood mathematics education. Mahwah, NJ: Lawrence Erlbaum Associates.

Clements, D. H. et Sarama, J. (2008). Experimental evaluation of the effects of a research-based preschool mathematics curriculum. American Educational Research Journal, 45(2), 443-494.

https://doi.org/10.3102/0002831207312908
Clements, D. H., Sarama, J., Wolfe, C. B. et Spitler, M. E. (2013). Longitudinal evaluation of a scale-up model for teaching mathematics with trajectories and technologies. American Educational Research Journal, 50(4), 812-850. https://doi.org/10.3102/0002831212469270

Cohen, J. (1988). Statistical power analysis for the behavioral sciences ( $2^{\mathrm{e}}$ éd.). Hillsdale, NJ : Erlbaum Associates.

Dehaene, S. (2011). The number sense: How the mind creates mathematics (édition révisée et mise à jour). New York, NY : Oxford University Press.

Deshaies, I. (2017). Effets d'une intervention didactique en mathématiques au préscolaire visant le développement $d u$ contrôle inhibiteur et adaptée au fonctionnement du cerveau sur l'apprentissage de préalables liés à l'arithmétique. Thèse inédite de doctorat, Université du Québec à Trois-Rivières.

Deshaies, I., Miron, J.-M. et Masson, S. (2015). Comprendre le cerveau des élèves pour mieux les préparer aux apprentissages en arithmétique dès le préscolaire A.N.A.E., 27(134), 39-45.

Deshaies, I., Miron, J.-M., Picard, C. et Masson, S. (2020). Mieux préparer les élèves du préscolaire à l'apprentissage de l'arithmétique: une recension des études proposant des programmes d'intervention s'appuyant sur les neurosciences. Neuroéducation, 6(1), 64-75. https://doi.org/10.24046/neuroed.202000601.64

De Vriendt, S. et Van Nieuwenhoven, C. (2010). L'enfant en difficulté d'apprentissage en mathématiques: Pistes de diagnostic et supports d'intervention. Marseilles, France : Solal.

Duncan, G. J., Dowsett, C. J., Claessens, A., Magnuson, K., Huston, A. C., Klebanov, P., ... et Japel, C. (2007). School readiness and later achievement. Developmental Psychology, 43(6), 1428-1446. https://doi.org/10.1037/0012-1649.43.6.1428

Fayol, M. (2008). L'acquisition de l'arithmétique élémentaire. Médecine/Sciences, 24(1), 87-90. https://doi.org/10.1051/medsci/200824187

Fayol, M. (2012). L'acquisition du nombre. Paris, France : Les Presses universitaires de France.

Fuchs, L. S. et Fuchs, D. (2005). Enhancing mathematical problem solving for students with disabilities. The Journal of Special Education, 39(1), 45-57. https://doi.org/10.1177/00224669050390010501

Fuson, K. (1991). Relations entre comptage et cardinalité chez les enfants de 2 à 8 ans. Dans J. Bideaud, C. Meljac et J.P. Fisher (dir.), Les chemins du nombre (p. 159-179). Lille, France : Les Presses universitaires de Lille.

Gallistel, C. R. et Gelman, R. (1992). Preverbal and verbal counting and computation. Cognition, 44(1-2), 43-74. https://doi.org/10.1016/0010-0277(92)90050-r 
Geary, D. C., Hamson, C. O. et Hoard, M. K. (2000). Numerical and arithmetical cognition: A longitudinal study of process and concept deficits in children with learning disability. Journal of Experimental Child Psychology, 77(3), 236-263. https://doi.org/10.1006/jecp.2000.2561

Geary, D. C., Hoard, M. K. et Bailey, D. H. (2012). Fact retrieval deficits in low achieving children and children with mathematical learning disability. Journal of Learning Disabilities, 45(4), 291-307. https://doi.org/10.1177/0022219410392046

Gelman, R. (1978). Counting in the preschooler: What does and does not develop? Dans R. S. Siegler (dir.), Children's thinking: What develops? (p. 213-241). Hillsdale, NJ : Erlbaum.

Gersten, R., Jordan, N. C. et Flojo, J. R. (2005). Early identification and interventions for students with mathematics difficulties. Journal of Learning Disabilities, 38(4), 293-304. https://doi.org/10.1177/00222194050380040301

Grégoire, J. et Van Nieuwenhoven, C. (1995). Counting at nursery school and at primary school: Toward an instrument for diagnostic assessment. European Journal of Psychology of Education, 10(1), 61-75. https://doi.org/10.1007/bf03172795

Griffin, S. (2004). Building number sense with number worlds: A mathematics program for young children. Early Childhood Research Quarterly, 19(1), 173-180. https://doi.org/10.1016/j.ecresq.2004.01.012

Houdé, O., Pineau, A., Leroux, G., Poirel, N., Perchey, G., Lanoë, C., ... et Mazoyer, B. (2011). Functional magnetic resonance imaging study of Piaget's conservation-ofnumber task in preschool and school-age children: A neo-Piagetian approach. Journal of Experimental Child Psychology, 110(3), 332-346.

https://doi.org/10.1016/j.jecp.2011.04.008

Houdé, O., Zago, L., Mellet, E., Moutier, S., Pineau, A., Mazoyer, B. et Tzourio-Mazoyer, N. (2000). Shifting from the perceptual brain to the logical brain: The neural impact of cognitive inhibition training. Journal of Cognitive Neuroscience, 12(5), 721-728.

https://doi.org/10.1162/089892900562525

Imbert, D. (2005). Pluralité des voies d'acquisition du comptage, ressources cognitives et acquisitions numériques. Thèse de doctorat inédite, Université de Nantes, France.

Kaufman, E. L., Lord, M. W., Reese, T. W. et Volkmann, J. (1949). The discrimination of visual number. The American Journal of Psychology, 62(4), 498-525. https://doi.org/10.2307/1418556

Klein, J. S. et Bizanz, J. (2000). Preschoolers doing arithmetic: The concepts are willing but the working memory is weak. Canadian Journal of Experimental Psychology, 54(2), 105-116. https://doi.org/10.1037/h0087333

Kohn, A. (2004). Feel-bad education : The cult of rigor and the loss of joy. Education Week, 24(3), 44-45.
Koontz, K. L. (1996). Identifying simple numerical stimuli: Processing inefficiencies exhibited by arithmetic learning disabled children. Mathematical Cognition, 2(1), 1-24. https://doi.org/10.1080/135467996387525

Landerl, K., Bevan, A. et Butterworth, B. (2004). Developmental dyscalculia and basic numerical capacities: A study of 8-9year-old students. Cognition, 93(2), 99-125. https://doi.org/10.1016/j.cognition.2003.11.004

Lépine, R., Camos, V. et Barrouillet, P. (2003). Différences individuelles chez l'enfant. Mémoire de travail et attention contrôlée. Dans A. Vom Hofe, H. Charvin, J. L. Bernaud et D. Guedon (dir.), Psychologie differentielle: recherches et réflexions (p. 227-231). Rennes, France: Presses universitaires de Rennes.

Lubin, A., Lanoë, C., Pineau, A. et Rossi, S. (2012). Apprendre à inhiber: une pédagogie innovante au service des apprentissages scolaires fondamentaux (mathématiques et orthographe) chez des élèves de 6 à 11 ans. Neuroéducation, 1(1), 55-84. https://doi.org/10.24046/neuroed.20120101.55

McGahee, T. W. et Tingen, M. S. (2000). The effects of a smoking prevention curriculum on fifth-grade children's attitudes, subjective norms and refusal skills. Southern Online Journal of Nursing Research, 1(2), 1-28.

Ministère de l'éducation, du loisir et du sport (MELS). (2003). Programme de formation à l'école québécoise. Éducation préscolaire. Enseignement primaire. Québec, QC: Gouvernement du Québec.

Ministère de l'éducation, du loisir et du sport (MELS). (2013). Projet de programme d'éducation préscolaire; maternelle 4 ans à temps plein en milieu défavorisé. Québec, QC: Gouvernement du Québec.

Mussolin, C., Mejias, S. et Noël, M.-P. (2010). Symbolic and nonsymbolic number comparison in children with and without dyscalculia. Cognition, 115(1), 10-25. https://doi.org/10.1016/j.cognition.2009.10.006

Noël, M.-P. (2005). La dyscalculie, trouble du développement numérique de l'enfant. Marseilles, France: Editions Solal.

Nosworthy, N., Bugden, S., Archibald, L., Evans, B. et Ansari, D. (2013). A two-minute paper-and-pencil test of symbolic and nonsymbolic numerical magnitude processing explains variability in primary school children's arithmetic competence. PLoS ONE, 8(7), e67918. https://doi.org/10.1371/journal.pone.0067918

Piazza, M., Facoetti, A., Trussardi, A. N., Berteletti, I., Conte, S., Lucangeli, D., ... et Zorzi, M. (2010). Developmental trajectory of number acuity reveals a severe impairment in developmental dyscalculia. Cognition, 116(1), 33-41. https://doi.org/10.1016/j.cognition.2010.03.012

Piazza, M., Izard, V., Pinel, P., Le Bihan, D. et Dehaene, S. (2004). Tuning curves for approximate numerosity in the human intraparietal sulcus. Neuron, 44(3), 547-555. https://doi.org/10.1016/j.neuron.2004.10.014 
Rossi, S., Lubin, A., Lanoë, C. et Pineau, A. (2012). Une pédagogie du contrôle cognitif pour l'amélioration de l'attention à la consigne chez l'enfant de 4-5 ans. Neuroéducation, 1(1), 29-54.

https://doi.org/10.24046/neuroed.20120101.29

Rourke, B. P. et Conway, J. A. (1997). Disabilities of arithmetic and mathematical reasoning. Perspectives from neurology and neuropsychology. Journal of Learning Disabilities, 30(1), 34-46.

https://doi.org/10.1177/002221949703000103

Rousselle, L. et Noël, M.-P. (2007). Basic numerical skills in children with mathematics learning disabilities: A comparison of symbolic vs non-symbolic number magnitude processing. Cognition, 102(3), 361-395. https://doi.org/10.1016/j.cognition.2006.01.005
Solomon, R. L. (1949). An extension of control group design. Psychological Bulletin, 46(2), 137-150.

https://doi.org/10.1037/h0062958

Sophian, C. (1998). A developmental perspective on child counting. Dans C. Donlan (dir.), The development of mathematical skills (p. 37-46). Hove, England : Psychology Press.

Van Nieuwenhoven, C., Grégoire, J. et Noël, M.-P. (2005). TEDI-MATH : Test diagnostique des compétences de base en mathématiques. Paris, France : Les éditions du centre de psychologie appliquée (ECPA).

Wilson, A. J., Revkin, S. K., Cohen, D., Cohen, L. et Dehaene, S. (2006). An open trial assessment of "The Number Race", an adaptive computer game for remediation of dyscalculia. Behavioral and Brain Functions, 2, 20. https://doi.org/10.1186/1744-9081-2-20 